\title{
HIV IN TUBERCULOSIS
}

\author{
Maj Gen R JAYASWAL*, Col PN ARORA+, \\ Lt Col BN PANDA\#
}

\begin{abstract}
This study was undertaken to evaluate clinical and laboratory parameters of 29 HIV-infected male patients with tuberculosis. Out of the 243 human immune deficiency virus (HIV) seropositive patients, 15 (6.2\%) were diagnosed to be suffering from tuberculosis whereas out of $\mathbf{3 5 0 2}$ cases of tuberculosis random HIV surveillance in 350 cases showed HIV infection in 14 (4\%). Mantoux positivity at the time of diagnosis of tuberculosis was noted in $16(55.1 \%)$ patients, and out of them $17.1 \%$ showed tuberculin conversion within 2 years of detection of HIV infection. Diagnosis of tuberculosis was based on demonstration of acid fast bacilli in sputum of $8(27.6 \%)$, and in lymphnode aspirate in $4(13.8 \%)$. Tubercular granuloma was demonstrated in 3 more patients, 2 in lymphnodes and one in liver parenchyma. In another 14 cases, there was classical clinical presentation and chest radiogram findings with excellent therapeutic response. Chest radiograms showed pulmonary infiltrate in 20, pleural effusion in 5, pleural thickening in 2, and intrathoracic adenopathy in 6 patients. Exclusive extrapulmonary tuberculosis was present in 4 patients.
\end{abstract}

MJAFI 1995; $51: 259-263$

KEY WORDS : HIV; Tuberculosis.

\section{Introduction}

$A$ $s$ the time passes, the old diseases not only show a change in their incidence but also in the pattern. Some diseases decline to the extent that they become almost extinct e.g., small pox. The change in the ecological balance brings in new diseases e.g., acquired immune deficiency syndrome (AIDS) [1]. AIDS has proved itself to be entirely different from all previously known diseases in that it affects not one organ or one system of organs but practically all the tissues of the human body. The background of AIDS leads to the deregulation of the cellular immune system which permits pathogenic activity on the part of opportunistic infections, infestations, and oncogenic agents. Recent reports confirm an increasing incidence of tuberculosis in HIV infected patients [1-3]. Pulmonary, and extrapulmonary (EPTB) tuberculosis, has been included in the definition of AIDS-defining criteria $[4,5]$ by Centre for Disease Control since 1993 and has also been accepted by Indian Council for Medical Research in Nov 94 [5]. The present study was designed to look for evidence of tuberculosis in HIV infected patients and for HIV seropositivity in patients of tuberculosis as well as to work out relevant clinical details.

\section{Material and Methods}

The material for the study included 15 patients of tuberculosis out of $243 \mathrm{HIV}$ seropositive patients hospitalized between Jan 1990 and Mar 1994; and 14 out of 3502 patients of tuberculosis hospitalized at Military Hospital (CTC) Pune who underwent HIV testing at random during the same period.

The methodology included detailed history regarding onset, clinical presentation, past history, family history, and history of contact with tuberculosis patients. General physical and systemic examination as regards to evidence of active or treated tuberculosis, other systemic diseases, cutaneous, and venereal diseases was carried out. Investigations

*Commandant, +Senior Adviser (Dermatology and Venereology). Command Hospital (SC); \#Classified Specialist (Medicine and Chest Diseases), Military Hospital (CTC), Pune - 411040. 
included estimation of haemoglobin, total and differential white cell count, erythrocyte sedimentation rate (Wintrobe's), urinalysis, blood sugar (fasting and postprandial), blood urea, serum creatinine, smear and culture examination of sputum for acid fast bacilli (AFB), Mantoux test, chest radiography, ELISA for HIV, fine needle aspiration cytology (FNAC) of lymphonode, skin biopsy for histopathological examination. scraping for fungus, staining and culture of pus discharge. and serological tests for syphilis as and when indicated. Western blot test was carried out for confirmation of HIV infection. Repeat investigations of prognostic value were carried out during follow up.

Mantoux test was carried out in all patients on detection of HIV antibody and repeated at 6 monthly intervals by using PPD ( 1 TU, RT-23 with Tween 80) obtained from BCG Vaccine Laboratory, Guindy, Madras. Reading was taken of mean induration (length + breadth/2) observed after 72 hours. Induration measuring $>5 \mathrm{~mm}$ was taken as positive for this study.

Diagnosis of tuberculosis was confirmed by demonstration of acid fast bacilli (AFB) in sputum or aspirate and/or demonstration of tubercular granuloma on histopathological examination. In the absence of confirmation, corroborative evidence were obtained by suggestive clinical, and radiological criteria supported by therapeutic response to antitubercular treatment (ATT).

\section{Results}

Twenty nine male patients were included in the study. Out of 243 HIV seropositive patients $15(6.2 \%)$ were diagnosed to be suffering from tuberculosis; whereas out of 3502 patients of tuberculosis, random HIV serosurveillance done in 350 revealed HIV infection in $14(4.0 \%)$ patients. The youngest patient was 20 years and the oldest was 53 years old. The age group most affected $(86.2 \%)$ was third and fourth decade of life. Out of them $\mathbf{8 2 . 8 \%}$ were married. History of extramarital sexual exposure were available in $69 \%$ patients. The mode of presentation was pyrexia of unknown origin in 18 cases ( $62.1 \%$ ), cough with expectoration in 1 , haemoptysis in 1 , diarrhea in 1 ( $3.5 \%$ each), loss of weight 7 $(24.1 \%)$, lymphadenopathy in $2(6.9 \%)$. Out of these 29 patients, 24 were transferred to us from various hospitals with initial suspicion/diagnosis of pulmonary and/or EPTB whereas 5 others were diagnosed to be having tubercular disease while they were undergoing routine evaluation after being detected HIV seropositive. They were not symptomatic for tuberculosis. Some patients had more than one presenting feature. Associated sexually transmitted diseases diagnosed during the period of hospitalization were present in $48.4 \%$. These were gonorrhea $(3.5 \%)$, chancroid $(13.8 \%)$ lymphogranuloma venereum $(3.5 \%)$, herpes genitalis $(6.9 \%)$, condylomata acuminata $(13.8 \%)$, and molluscum contagiosum $(6.9 \%)$. Cutaneous and $\mathrm{mu}$ cocutaneous disorders were noted in $38.1 \%$,

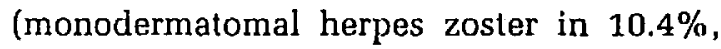
disseminated herpes zoster in $3.5 \%$, mucocutaneous candidiasis in $6.9 \%$, and seborrhoeic dermatitis in $10.4 \%$ ). Viral hepatitis, reactive depression and kala-azar were present in one patient each. Opportunistic infection with crytococcus in 3 and cytomegalovirus infection in 1 patient were noted.

Hemoglobin was less than $10 \mathrm{gm} \%$ in $6.9 \%, 10-14 \mathrm{~g} \%$ in $79.3 \%$ and more than 14 $\mathrm{g} \%$ in $13.8 \%$. ESR was $10 \mathrm{~mm}$ fall after $1 \mathrm{st}$ hour (Wintrobe's) in $27.6 \%, 11-20 \mathrm{~mm}$ fall in $37.9 \%, 21$ to 30 in $6.9 \%, 31$ to 40 in $10.4 \%$ and $4 \mathrm{~mm}$ fall in the remaining $17.2 \% \mathrm{pa}-$ tients. $\mathrm{HBsAg}$ was positive in $10.4 \%$. Mantoux test was non-reactor in $55.1 \%$, less than $5 \mathrm{~mm}$ in $13.8 \%$, more than $5 \mathrm{~mm}$ in $3.5 \%$, more than $10 \mathrm{~mm}$ in $17.2 \%$, more than $15 \mathrm{~mm}$ in $6.9 \%$ and more than $20 \mathrm{~mm}$ in the remaining $3.5 \%$. Out of total patients $17.2 \%$ converted to Mantoux positive during the posthospital follow up of FIIV, one each after 6,12 and 18 months and 2 after 24 months. Sputum for AFB was positive in 8 cases. Fine needle aspiration of lymphnode was done in $31 \%$ cases, $4(13.8 \%)$ revealed AFB. Lymphnode biopsy done in 3 patients revealed tubercular pathology in 2 patients and normal pathology 
in 1. Radiological examination revealed parenchymatous lesions in 20 (minimal in 17 , moderately advanced in 2 and far advanced in 1), pleural effusion in 5 , pleural thickening in 2, hilar lymphadenopathy in 5, and paratracheal lymphadenopathy in 1 . The patients were treated for 2 months with streptomycin (S) or ethambutol (E) with rifampicin (R), isonex $(\mathrm{H})$ and pyrazinamide $(\mathrm{Z})$ followed by $\mathrm{RH}$ for 10 months. All of them responded well to therapy with sputum conversion, weight gain and good radiological regression.

Extra pulmonary involvement included pleural disease in 7 , extra-thoracic lymphnode tuberculosis in 5 , pericardial effusion in 2. hepatic granuloma in 1 and mesenteric adenopathy with exudative ascites in 1 patient.

Associated infections included 3 cases with disseminated cryptococosis and of these 2 cases were confirmed on autopsy. Though both these cases had antemortem bacteriological confirmation of tubercular disease, subsequent pulmonary lesions were caused by disseminated cryplococcus infection. One patient showed cytomegalovirus retinitis.

Most lymph node biopsy aspirates showed AFB without marked caseating granuloma or giant cells, a peculiarity known to occur in HIV positive cases $[3,6,7]$.

\section{Discussion}

In June 1981, the first report was published on the occurrence of certairi opportunistic diseases that we have since learned to consider a characteristic part of the acquired immune deficiency syndrome caused by HIV infection $[3,8]$. Over the 14 years that have intervened between that time and the present, the few cases initially reported have grown into a pandemic. Tuberculosis is a frequent opportunistic infection and may be one of the mode of presentation of the disease syndrome [8]. This is of particular importance because it is contagious by the respiratory route, readily treatable and potentially preventable with chemoprophylaxis. However, the degree of overlap in a population will determine the impact of HIV on tuberculosis.
WHO estimates that 8 to 10 million new cases of tuberculosis occur every year, three fourths being in tropical countries [9]. The incidence of tuberculous infection is the highest between 5 to 20 years of age [2] rising with and reaching maximum of $70 \%$ by 35 years of age. The age groups maximum affected in the present series were 3rd and 4th decade of life. The later age appears to be affected because the study included only Armed Forces personnel who join service as healthy individuals after medical screening around the age of 20 years and it is subsequent to that they acquired the disease. The commonest route of transmission of HIV infection is by sexual means. Of the total patients, $69.0 \%$ had volunteered history of pre/extramarital sexual exposure. It is likely that in some of them, after having acquired HIV infection, opportunistic tubercular infection in general population had affected the individuals under the present study. With $30 \%$ of general population of this country being already infecled with tuberculosis [10] the chances of reactivation of dormant foci of infection remains high. However it is also being increasingly felt that HIV infected individuals can also rapidly develop disease from recently acquired infection [7].

The mode of presentation of tuberculosis in our series was pyrexia of unknown origin in $62.1 \%$. Sputum positivity was noted in $27.5 \%$ cases. This positivity is less than that of Mohanty et al [10] but it is also much less than the usial sputum positivity found in tuberculosis patients from the service population [11]. This supports the known concept that HIV positive patients are more often sputum negative and they are less likely to have extensive disease $[3,7]$ in comparison to usual picture in HIV negative tuberculosis patients [11] in our setup.

It is estimated that incidence of tuberculosis in patients with HIV infection is almost 100 to 500 times the incidence in general population [12]. Mantoux positivity (>5 mm) of $31.1 \%$ is definitely less than that of ICMR, Pune based study which showed positivity of $51 \%[7]$. Induration of $10 \mathrm{~mm}$ or more is the 
criteria for a positive tuberculin test in most clinical situations, whereas $>5 \mathrm{~mm}$ is considered positive in patients with HIV infection, because their delayed type hypersensitivity response is reduced [3]. Although a positive tuberculin skin test increases the likelihood of tuberculosis, a negative test does not exclude the diagnosis. The positivity of results of at least $10 \mathrm{~mm}$ in diameter was $44.9 \%$. Out of the remaining $55.1 \%$ Mantoux negative, $17.2 \%$ converted to Mantoux positive during the six-monthly follow up within 2 years. This could be due to fresh infection which has resulted in Mantoux conversion. The remaining continued to remain Mantoux negative possibly because of advanced stage of HIV induced immunosuppression. Available active T-lymphocytes are likely to be already overwhelmed by mycobacterial antigens at the focus of infection thus not being available for producing demonstrable positive reaction at tuberculin test site. Further tests to confirm skin anergic state could not be performed due to lack of facilities. In the present study, sputum for AFB was positive in $27.6 \%$. Another $13.8 \%$ were diagnosed to be AFB positive on FNAC of lymphnodes (out of these $3.4 \%$ were sputum positive as well). Histopathology consistent with tuberculosis was observed in $17.8 \%$ cases of lymph node biopsy. An interesting finding recorded was that giant cells and characteristic tubercular granulomas were uncommon.

The chest radiogram manifestations of pulmonary tuberculosis correlate with the degree of HIV induced immunosuppression. Chest radiographic findings are often similar to those of reactivation tuberculosis in immunocompetent patients, including cavitation and upper lobe infiltrates, among patients, including cavitation and upper lobe infiltrates, among patients with relatively well preserved immune function. In contrast, among more severely immunosuppressed patients with HIV infection the chest radiographic finding are typically those of tuberculosis in immunocompetent patients such as hilar adenopathy, pleural effusions and a miliary pattern. Infiltrates may involve upper and lower lung fields. Hilar adenopathy, pleural effusion, and cavitation are of great diagnostic value since these are rarely features of $P$. carinii pneumonia [6]. In the present study radiological examination revealed parenchymatous lesions in $58.7 \%$, pleural involvement in $24.2 \%$, hilar and paratracheal lymphadenopathy in $17.4 \%$. Abdominal tuberculosis was diagnosed in $3.5 \%$ and disseminated tuberculosis in $10.4 \%$.

The response to antitubercular treatment has been reported to be good in patients with HIV infection. Ideal duration remains to be determined. As per CDC, therapy should be continued for at least 9 months and at least six months beyond sputum culture negativity [12]. Patients with extrapulmonary tuberculosis should be treated for at least 12 to 18 months. Therapeutic schedules followed in the present study were $2 \mathrm{EHRZ}+10 \mathrm{HR}$ with satisfactory response in all in contrast to observation of Mohanty et al [10] where poor response to therapy was noted in tuberculin negative patients. However, after initial control of tuberculous infection two of them developed fungal infection leading to fatality.

The associated diseases recorded in HIV seropositive tuberculosis patients were viral hepatitis, kala-azar, reactive depression, and cytomegalovirus infection in $3.5 \%$ each, cryptococcal infection and mucocutaneous candidiasis in $6.9 \%$ each, seborrhoeic dermatitis in $10.4 \%$, herpes zoster in $13.8 \%$ and STD in $48.4 \%$ patients. Two patients died. Postmortem revealed disseminated cryptococcosis. However, there was no existing tubercular granuloma in both cases though AFB was seen in sputum in one and lymph node in the other at the time of diagnosis. This seems to be an indicator that all these patients respond well to antitubercular chemotherapy. These associations, except kala-azar, had been recorded by other workers $[13,14]$.

It was recorded in the present study that $6.2 \%$ out of 243 HIV seropositive patients were diagnosed to be suffering from tuberculosis, whereas random HIV serosurveillance of 350 cases of tuberculosis revealed HIV 
infection in $4.0 \%$. Pulmonary tuberculosis was the most common manifestation of tuberculous disease in HIV positive infection, disease was usually confined to lung, whereas when tuberculosis was diagnosed after the onset of HIV infection the chances of extrapulmonary tubercular infections were enhanced.

To conclude, HIV infection is an important risk factor for resurgence of tuberculosis. Reemergence of tuberculosis as a major public health problem can have sinister significance in future for a developing country like India where tuberculosis is endemic and HIV infection is spreading rapidly. This demands increased vigilance and quadrupled effort to combat the dual pandemic.

\section{REFERENCES}

1. Arora PN, Jha PK, Das AL.Changing trends of STD in Armed Forces. Medical Journal Armed Forces India 1993; 49 : 91-4.

2. WHO. Current and future dimension of HIV/AIDS pandemic : A capsule summary. 1992; 1-15.

3. Sivaraman V, Furnandez G, Rao PS. HIV infection and pulmonary tuberculosis. Indian J Tuberculosis 1992; $30: 35-9$.

4. CDC 1993. Revised classification system for HIV infection and expanded surveillance case dofinition for AIDS among adolescents and adults. MMWR 1993; $41: 1-15$.

5. ICMR Bullotin : AIDS and Family 1994; $24: 115-30$.

6. Modilevsky T, Sattler FR, Barnes PF. Mycobacterial discase in patients with human immunodeficiency virus infection. Arch Intern Mod 1989; 149 : 2201-5.

7. Sharma SK, Pande JN. HIV infection and tuberculosis. Ind J Chest Dis Allied Sci 1994; 36 : 101-11.

8. Arora PN. Modes of presentation of HIV infection. Indian J Sexually Transmitted Diseases 1993; 14 : 46-9.

9. Park JE, Park K. Text book of preventive and social medicine. 12th ed. 1989.

10. Mohanty KC, Nair S, Sahasrabudhe T. Changing trends of HIV infection in patients of respiratory disease in Bombay since 1988. Indian J Tuberculosis 1994; 41 : 147-50.

11. Jayaswal R, Panda BN, Debnath J. Three short-course chemotherapy for pulmonary tuberculosis in Armed Forces: A comparison. Lung India 1991; 47 : 104-7.

12. Tuberculosis and human immunodeficiency virus infection : Recommondation of the advisory committee for the elimination of tuberculosis. MMWR 1989; $38: 236-50$.

13. Ansari MA, Hira SK, Bayley AC. A colour atlas of AIDS in tropics. London : Wolfe Medical Publication, 1989; 18-28.

14. Greanspan JS, Barr CE, Seubba JJ. Oral manifestations of HIV infection. Oral Surg Oral Med Oral Pathol 1992; 142-4. 